\title{
Electron transport and magnetic properties of the filled skutterudite compound $\mathrm{ThFe}_{4} \mathrm{As} 12$
}

\author{
R. Wawryk*, O. Żogał, A. Rudenko, T. Cichorek, and Z. Henkie \\ Institute of Low Temperature and Structure Research, Polish Academy of Sciences, P.O. Box 1410, 50-950 Wrockaw \\ 2, Poland \\ M. B. Maple \\ Department of Physics and Center for Advanced Nanoscience, University of California, San Diego, La Jolla, \\ California 92093 USA
}

Keywords: Semimetals, transport properties, two-band model, charge carrier density and mobility

\begin{abstract}
$\underline{\text { Abstract }}$
Single crystals of the filled skutterudite compound $\mathrm{ThFe}_{4} \mathrm{As}_{12}$ were examined down to $T=0.4 \mathrm{~K}$ and in magnetic fields up to $B=9 \mathrm{~T}$. Electron transport properties combined with results of Hall-effect measurements indicate semimetallic properties with a charge carrier density of the order of $2 \times 10^{20} \mathrm{~cm}^{-3}$. In terms of a two-band model, the effective mass of the electrons is nearly a factor of 5 larger than the hole effective mass. A small carrier density is in line with the lack of magnetic ordering in this Fe-based filled skutterudite. Therefore, a Sommerfeld coefficient of the electronic specific heat of $45 \mathrm{mJmol}^{-1} \mathrm{~K}^{-2}$ for semimetallic $\mathrm{ThFe}_{4} \mathrm{As}_{12}$ is an unexpected observation whose origin remains to be explained.
\end{abstract}

\section{Introduction}

The ternary transition metal pnictides with the chemical formula $M T_{4} P n_{12}(M=$ alkali metal, alkaline earth, lanthanide or actinide; $T=\mathrm{Fe}, \mathrm{Ru}, \mathrm{Os} ; P n=\mathrm{P}, \mathrm{As}, \mathrm{Sb}$ ), which crystallize in the filled skutterudite structure (space group Im-3), exhibit a wide variety of strongly correlated electron phenomena [1-4]. Many of these phenomena depend on hybridization between the rare earth or actinide $f$-electron states and the conduction electron states which, in some filled skutterudite systems, leads to the emergence of semiconducting behavior. This trend is evident in the cerium-filled skutterudite systems, most of which are semiconductors where the gap size is correlated with the lattice constant [5-7].

Previous studies have shown a semiconducting ground state in all the Ce-filled arsenide skutterudites [8-11]. However, it turned out that low-temperature properties of $\mathrm{CeFe}_{4} \mathrm{As}_{12}$ and $\mathrm{CeRu}_{4} \mathrm{As}_{12}$ are strongly sample dependent: For the latter system, polycrystalline samples display semiconducting behavior with an energy gap of $50 \mathrm{~K}$, [8] while single crystals have a metallic ground state and exhibit non-Fermi-liquid behavior [9].

*Corresponding author: R.Wawryk@int.pan.wroc.pl 
Intriguingly, for $\mathrm{CeFe}_{4} \mathrm{As}_{12}$, semiconducting vs. metallic properties depend on morphology of single-crystalline specimens [10]. Contrary to these systems, an increase of the electrical resistivity of $\mathrm{CeOs}_{4} \mathrm{As}_{12}$ by two orders of magnitude upon cooling below about $135 \mathrm{~K}$ is robust against sample variation [11]. For all the $\mathrm{Ce}_{4} \mathrm{As}_{12}$ filled skutterudites, however, magnetic susceptibility measurements signal a nonmagnetic state at low temperatures. Such a property of Ce-based materials can be described by one of two well-known models: The first is the intermediate valence picture where the Ce ions have a dynamic temporally fluctuating valence between $3+$ (magnetic) and $4+$ (nonmagnetic), which evolves into a nonmagnetic ground state below a characteristic valence fluctuation temperature $T_{\mathrm{vf}}[12,13,14]$. The second model involves a Kondo effect [15] where the localized magnetic moments of $\mathrm{Ce}^{3+}$ ions, which occupy a sublattice, are screened by conduction electrons, resulting in a nonmagnetic ground state, below a characteristic Kondo temperature $T_{\mathrm{K}}$. It should be emphasized that in the valence fluctuation model or Kondo-effect scenario an opening of a narrow energy gap at the Fermi level is predicted. Most likely, such Kondo physics is realized in $\mathrm{CeOs}_{4} \mathrm{As}_{12}$ where the residual resistivity is reduced by more than a factor of 10 with $B=5 \mathrm{~T}$ at $T<3 \mathrm{~K}[11,16]$.

For completeness, we note that in the series $M \mathrm{Fe}_{4} \mathrm{As}_{12}$ with $M$ being $\mathrm{La}, \mathrm{Ce}, \mathrm{Pr}, \mathrm{Nd}, \mathrm{Sm}$, and $\mathrm{Eu}[17,18$, 19], magnetism due to itinerant $\mathrm{Fe}-3 d$ electrons is only not observed in $\mathrm{CeFe}_{4} \mathrm{As}_{12}$, as mentioned above. In all other $M \mathrm{Fe}_{4} \mathrm{As}_{12}$ systems with localized $4 f$ electrons, enhanced Curie temperatures $T_{\mathrm{C}}$ and magnetization compared to those of metallic $\mathrm{LaFe}_{4} \mathrm{As}_{12}$ (itinerant ferromagnetism with $T_{\mathrm{C}}=5.2 \mathrm{~K}$ [20] vs $3.8 \mathrm{~K}$ [21]) indicate a cooperative contribution to the ferromagnetism from $\mathrm{Fe} 3 d$ and localized $4 f$ magnetic moments.

This paper addresses transport and magnetic properties as well as the specific heat, measured as a function of temperature and applied magnetic field, of the filled skutterudite compound $\mathrm{ThFe}_{4} \mathrm{As}_{12}$. Based on the fact that Th is an element with the fixed +4 oxidation state, we show that this material should be regarded as a semimetal. Thus, our findings for $\mathrm{ThFe}_{4} \mathrm{As}_{12}$ should be relevant to the class of $\mathrm{Ce} T_{4} \mathrm{Pn}_{12}$ systems with semiconducting properties.

These compounds were derived from the binary pnictides $T X_{3}$, the prototype of which is $\mathrm{CoAs}_{3}$, a diamagnetic zero gap semiconductor [22]. The unit cell of $\mathrm{CoAs}_{3}$ is a cube formed with 8 octants, the corners of which are occupied by $\mathrm{Co}^{3+}$ cation atoms. The centers of six of the octants are filled by square radicals $\left[\mathrm{As}_{4}\right]^{4-}$, while the two remaining octants are empty. Therefore, the valence ratio Co:[As 4 is equal to $4: 3$ and the total structure remains electrically neutral, favoring a semiconducting electronic structure. Thus, the $T X_{3}$ compounds belong to the family of Zintl phases; i.e., solid compounds of metals with nonmetals or metalloids forming polyanions [23]. According to the Zintl concept, 24 valence electrons per $T X_{3}$ formula, or 96 valence electrons per $M T_{4} X_{12}$, stabilize such skutterudite compounds, yielding a preference for semiconducting behavior. Following this concept, Luo et al. [24] have discovered a large family of filled skutterudites, based on the group 9 transition metals Co, Rh, and Ir, the alkali, alkaline-earth, and rare-earth elements and $\left[\mathrm{Sb}_{4}\right]^{4-}$ polyanions. Moreover, electronic structure calculations - for some representatives of this group - showed they are semiconductors. Replacing Fe (as well as by Ru or Os) with Co, $\left[\mathrm{As}_{4}\right]^{4-}$ with $\left[\mathrm{Sb}_{4}\right]^{4-}$ polyanions, and filling the two empty octants by Th or tetravalent Ce leaves the structure electrically neutral with the 4:3 valence ratio and 96 valence electrons. Therefore, the semiconducting or 
semimetallic properties are expected for the modified skutterudites. This applies to the properties of the phosphide and antimonide skutterudites, as well. Indeed, $\mathrm{CeOs}_{4} \mathrm{As}_{12}$ [11], $\mathrm{CeRu}_{4} \mathrm{As}_{12}[8,9]$, and $\mathrm{CeFe}_{4} \mathrm{As}_{12}$ [10] become semiconductors at low temperatures with the highest thermoelectric power observed for $\mathrm{CeFe}_{4} \mathrm{As}_{12}$. The latter observation is for a sample that has off-stoichiometry defects of order $10^{20} \mathrm{~cm}^{-3}$ with a maximum thermoelectric power $+86 \mu \mathrm{V} / \mathrm{K}$ at $120 \mathrm{~K}$. The $\mathrm{Ce}$ and $\mathrm{U}$ iron phosphides filled skutterudites, $\mathrm{CeFe}_{4} \mathrm{P}_{12}$ and $\mathrm{UFe}_{4} \mathrm{P}_{12}$, were also found to be semiconductors. In the aforementioned compounds, presumably the tetravalent states of the filler atoms and the semiconducting state are a result of the hybridization of one $f$-electron of Ce [25] or $\mathrm{U}$ [26]. Accordingly, an energy gap in $\mathrm{CeFe}_{4} \mathrm{P}_{12}$ [27] and semimetallic state for $\mathrm{UFe}_{4} \mathrm{P}_{12}[28,29]$, have been anticipated by electronic band structure calculations. On the other hand, when the iron phosphide filler ion is nonmagnetic tetravalent thorium, we obtain $\mathrm{ThFe}_{4} \mathrm{P}_{12}$ whose electrical resistivity exhibits a monotonic temperature dependence of metallic character [30]. The metallic behavior has also been confirmed in infrared reflectance spectroscopy measurements [25] and tightbinding calculations of the electronic structure [29]. In contrast, a semiconducting state with a direct gap has been predicted (for $\mathrm{ThFe}_{4} \mathrm{P}_{12}$ ) by electronic band structure calculations performed by Takegahara and Harima [31], Khenata et al. [27] and an indirect gap by Cheng et al. [32]. The $\mathrm{CeFe}_{4} \mathrm{As}_{12}$ (small gap semiconductor), in turn, exhibits semimetallic properties when (unintentionally) heavily doped [10].

\section{Experimental Details}

Single crystals of $\mathrm{ThFe}_{4} \mathrm{As}_{12}$, with dimensions up to $1 \mathrm{~mm}$, were grown from elements with purities > $99.9 \%$, by synthesis and mineralization in a molten Cd:As flux at high temperatures and pressures, as described previously [33]. The elemental components were combined in the atomic ratio Th:Fe:Cd:As = 1:4:12:48 $\left(\mathrm{ThFe}_{4} \mathrm{As}_{12}\right.$ $+12 \mathrm{CdAs}_{3}$ ). The starting materials were sealed in pyrolised quartz ampoules, heated to $820{ }^{\circ} \mathrm{C}$ at a rate of $20{ }^{\circ} \mathrm{C} / \mathrm{hr}$, and repeatedly heated/cooled between 820 and $780{ }^{\circ} \mathrm{C}$ for six weeks at a rate of $3{ }^{\circ} \mathrm{C} / \mathrm{hr}$. Finally, the ampoules were cooled to room temperature at a rate of $20^{\circ} \mathrm{C} / \mathrm{h}$. The heating was done in a home built high pressure cell filled with $19 \mathrm{~atm}$ of $\mathrm{Ar}$, which is approximately equal to the estimated vapor pressure of the $\mathrm{CdAs}_{3}$ flux in the ampoule at 820 ${ }^{\circ} \mathrm{C}$. The main purpose of the $\mathrm{Ar}$ is to balance the pressures inside and outside the inner ampoule and thereby avoid an explosion. The solidified flux was removed from $\mathrm{ThFe}_{4} \mathrm{As}_{12}$ crystals by sublimation in high vacuum at $600{ }^{\circ} \mathrm{C}$. The crystals were cleaned in acid to remove a possible cerium arsenide impurity phase from the surface of the crystals.

Several $\mathrm{ThFe}_{4} \mathrm{As}_{12}$ crystals were ground into a powder for X-ray diffraction (XRD) measurements at room temperature with an X'pert Pro PANanalitical diffractometer with $\mathrm{Cu} \mathrm{K} \alpha$ radiation. The crystal structure refinements and the theoretical XRD pattern calculations were done applying the X'pert High Score Plus program and the $\mathrm{LaFe}_{4} \mathrm{As}_{12}$ pattern as the reference.

Electron transport properties of the $\mathrm{ThFe}_{4} \mathrm{As}_{12}$ single crystals were investigated on six different samples. For all of them, the thermoelectric power was estimated at room temperature. For the most representative specimens, the thermoelectric power was measured down to $0.4 \mathrm{~K}$. The electrical resistivity, magnetoresistance, and Hall effect were studied by a standard four-point $\mathrm{AC}$ technique in the temperature range $2 \mathrm{~K} \leq T \leq 300 \mathrm{~K}$ and in applied 
magnetic fields up to $9 \mathrm{~T}$ using a ${ }^{3} \mathrm{He}$ cryostat. DC magnetization measurements were performed for $1.8 \mathrm{~K} \leq T \leq 300$ $\mathrm{K}$ and in varying magnetic fields up to $5.5 \mathrm{~T}$ utilizing a superconducting quantum interference device magnetometer (MPMS, Quantum Design). The magnetization was investigated on two different specimens with approximately the same mass of $400 \mathrm{mg}$. The first was an ensemble of dozens of large single crystal and the second was composed of few hundred tiny single crystals. Specific heat measurements in the temperature range $0.4 \mathrm{~K} \leq T \leq 10 \mathrm{~K}$ were performed on a few single crystals with a mass of $16.5 \mathrm{mg}$ via the thermal-relaxation method using a commercial ${ }^{3} \mathrm{He}$ microcalorimeter (PPMS, Quantum Design).

\section{Experimental results}

The X-ray powder diffraction pattern, which contains no impurity phase peaks, reveals that the single crystals consist of single phase $\mathrm{ThFe}_{4} \mathrm{As}_{12}$. The pattern was identified with the $\mathrm{LaFe}_{4} \mathrm{P}_{12}$-type structure (Im $\overline{3}$ space group) with two formula units per unit cell. Figure 1 shows the powder XRD pattern for quantitative Rietveld structure refinement of several powdered $\mathrm{ThFe}_{4} \mathrm{As}_{12}$ single crystals. There is good agreement between the observed and calculated pattern for stoichiometric $\mathrm{ThFe}_{4} \mathrm{As}_{12}$ skutterudite (Th:Fe:As = 1:4:12). The cubic crystallographic cell parameter determined by the last squares fitting to all observed reflections using profile fit is $a=8.2970(6) \AA$. Atomic coordinates $\mathrm{x}, \mathrm{y}$, and $\mathrm{z}$ are equal to $0,0,0$ for Th, and $0.25,0.25,0.25$ for Fe. For As these coordinates are: $\mathrm{x}=0.1549(4), \mathrm{y}=0.3447(3)$, and $\mathrm{z}=0.000$.

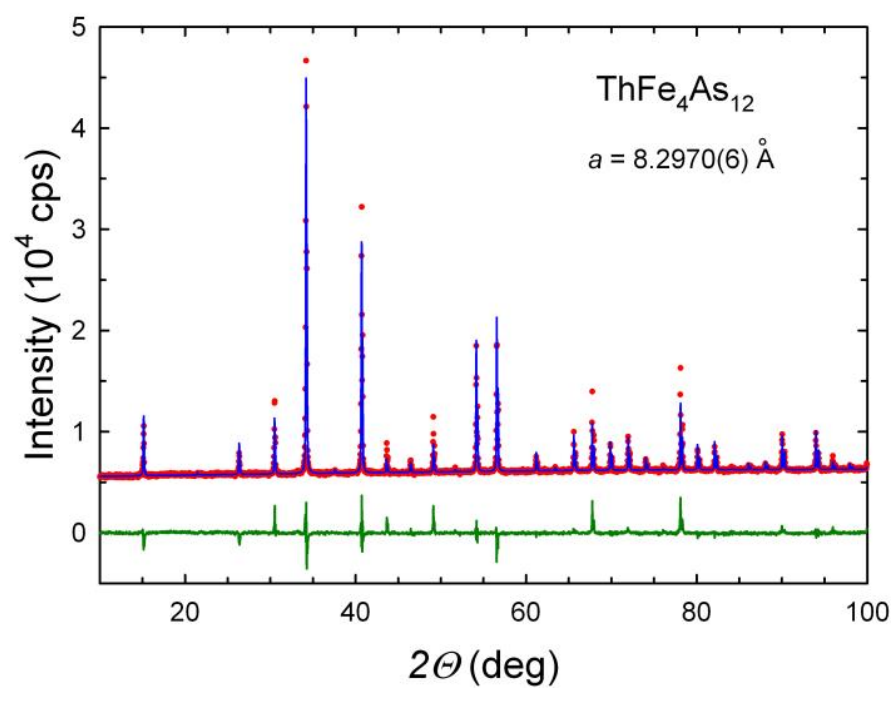

Fig. 1. Rietveld refinement for $\mathrm{ThFe}_{4} \mathrm{As}_{12}$ skutterudite. Red points are observed data; the upper (blue) curve is the calculated pattern; the lower (green) curve is the difference between observed and calculated diffraction pattern. Refined structural parameters are included in the text.

The discrepancy factor $\mathrm{R}$ (weighted profile) equals to $3 \%$. The obtained here lattice parameter $a$ is close to value 8.314(2) $\AA$ determined from $\mathrm{ThFe}_{4} \mathrm{As}_{12}$ single crystal diffraction [33].

The temperature dependence of the magnetic susceptibility $\chi$ is shown in Fig. 2. In this case, $\chi$ is defined as $\chi \equiv \partial M / \partial H$, instead of $M / H$, in order to eliminate a small nonlinear contribution to the magnetization $(M)$ (see inset Fig. 2) to obtain the intrinsic magnetization. 


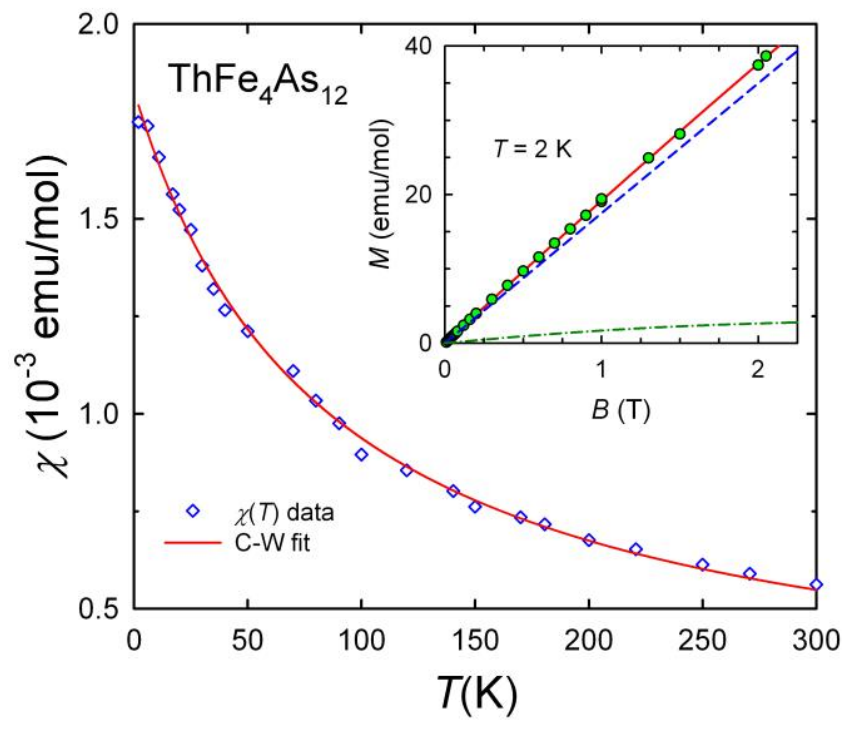

Fig. 2. Temperature dependence of the magnetic susceptibility $\chi$, taken as the initial slope of the magnetization vs. magnetic field at each temperature. The solid line represents a fit to the MCW function (Eq. (1)) Inset: Magnetic field dependence of the magnetization at $2 \mathrm{~K}$ (circle and solid line) and its decomposition into linear (dashed line) and Brillouin function (dash-dotted line) components.

impurities (or phases) that were not detectable in the XRD patterns.

The $\chi(T)$ data obey a modified Curie-Weiss (MCW) law, $\chi(T)=C /(T-\Theta)+\chi_{0}$ with a negative Curie-Weiss temperature $\Theta=-83.7 \mathrm{~K}$. Using $C=N_{\mathrm{A}} \mu_{\mathrm{eff}}^{2} / k_{\mathrm{B}}$, where $N_{\mathrm{A}}$ is Avogadro's number and $k_{\mathrm{B}}$ is the Boltzmann constant, one obtains the paramagnetic effective moment $\mu_{\mathrm{eff}}=1.05 \mu_{\mathrm{B}} / f . u$. and $\chi_{0}=1.91 \times 10^{-4}(\mathrm{emu} / \mathrm{mol})$. The measured total magnetic susceptibility per mole of $\mathrm{ThFe}_{4} \mathrm{As}_{12}$ can be partitioned as

$$
\begin{gathered}
\chi(T)=\chi_{\mathrm{d}}(T)+\chi_{0} \\
\chi_{\mathrm{d}}(T)=\chi_{\mathrm{Th} 6 d}(T)+4 \chi_{\mathrm{Fe} 3 d}(T) \\
\chi_{0}=\chi_{\mathrm{Th}-\mathrm{orb}}+\chi_{\mathrm{Th}-\mathrm{dia}}+4\left(\chi_{\mathrm{Fe}-\mathrm{orb}}+\chi_{\mathrm{Fe}-\mathrm{dia}}\right)+12 \chi_{\mathrm{As}}
\end{gathered}
$$

where $\chi_{\text {dia }}, \chi_{\text {orb}}$, and $\chi_{\mathrm{d}}$ are the diamagnetic, $6 d(3 d)$ - orbital, and $6 d(3 d)-$ spin contributions from Th(Fe) sites, respectively. Finally, $\chi_{\text {As }}$ represents the susceptibility of the As atoms. Thus, a main component of the magnetic susceptibility is the spin-dependent contribution $\chi_{\mathrm{d}}(T)$, discussed later. The temperature-independent magnetic susceptibility, $\chi_{0}$ includes the contributions of core electron diamagnetism and Van Vleck terms. The simplified and crude form for the latter is given by equation (2) [34],

$$
\chi^{\mathrm{Vv}} \approx \frac{4 N_{\mathrm{A}} \mu_{\mathrm{B}}^{2} N_{\mathrm{o}} N_{\mathrm{u}}}{\left(N_{\mathrm{o}}+N_{\mathrm{u}}\right) \Delta}
$$

where $\mu_{\mathrm{B}}$ is the Bohr magneton, $N_{\mathrm{o}}$ and $N_{\mathrm{u}}$ are numbers of occupied and unoccupied states in the conduction band, for $3 d$ and $6 d$ electrons $N_{\mathrm{o}}+N_{\mathrm{u}}=10$, and $\Delta$ is a mean separation of the occupied and unoccupied $3 d$ and $6 d$ subbands. In the case of $\mathrm{LaFe}_{4} \mathrm{As}_{12}, \chi_{0}$ is negligibly small, as compared to $\chi(300 \mathrm{~K})=13 \times 10^{-4}(\mathrm{emu} / \mathrm{mol})$, while substitution of 
$\mathrm{Th}^{4+}$ for $\mathrm{La}^{3+}$ enhances $\chi_{0}$ to the value $1.91 \times 10^{-4}(\mathrm{emu} / \mathrm{mol})$. Diamagnetic contributions of $\mathrm{Th}^{4+}$ substitutions for $\mathrm{La}^{3+}$ cations to the susceptibility of corresponding skutterudites are equal to -0.23 and $-0.20\left(10^{-4} \mathrm{emu} / \mathrm{mol}\right)$ [35], respectively. Thus, instead of a small Van Vleck term as expected for the filled $3 d$ and $6 d$ levels in anticipated semiconducting and diamagnetic properties of $\mathrm{ThFe}_{4} \mathrm{As}_{12}$, we observe a sizable contribution $\chi_{0}$ to $\chi$, suggesting incompletely filled $3 d\left(\mathrm{Fe}^{+3}\right)$ and/or empty $6 d\left(\mathrm{Th}^{+4}\right)$ levels and a non-semiconducting (metallic or semimetallic) state.

The zero-field low-temperature dependence of the specific heat $C(T)$ of $\mathrm{ThFe}_{4} \mathrm{As}_{12}$ is shown in Fig. 3. Here, we have assumed that the $2 a$ position in the filled skutterudite structure is fully occupied by the Th atoms. The $C(T)$ data do not show any anomalies down to $0.4 \mathrm{~K}$ and remain virtually the same in a magnetic field of $9 \mathrm{~T}$ (not presented). As for other filled skutterudites (see e.g.[36]), the lattice heat capacity of $\mathrm{ThFe}_{4} \mathrm{As}_{12}$ can be modeled by a combination of an Einstein mode $C_{\mathrm{E}}$ associated with the filler Th atom and the usual Debye term $C_{\mathrm{D}}$ due to the remaining 16 atoms of the polyanion $\left[\mathrm{Fe}_{4} \mathrm{As}_{12}\right]$. Thus, the $C(T)$ results in the temperature range $0.4-10 \mathrm{~K}$ can be expressed as $C(T)=\gamma T+C_{\mathrm{D}}+C_{\mathrm{E}}$. Here, the Debye temperature was fixed to $370 \mathrm{~K}$ in accord with $\Theta_{\mathrm{D}}=373 \mathrm{~K}$ for $\mathrm{NdFe}_{4} \mathrm{As}_{12}$ [37]. A least-squares fit yields $\gamma=45(1) \mathrm{mJ} / \mathrm{mol}-\mathrm{K}^{2}$ and the Einstein temperature $\Theta_{\mathrm{E}}=86(1) \mathrm{K}$ (cf. dashed lines in Fig. 3).

Note that the Sommerfeld coefficient of the electronic specific heat of $\mathrm{ThFe}_{4} \mathrm{As}_{12}$ is about one order of magnitude larger than $\gamma=3.3 \mathrm{~mJ} / \mathrm{mol}-\mathrm{K}^{2}$ of semiconducting and nonmagnetic $\mathrm{CeFe}_{4} \mathrm{As}_{12}$ [10]. On the other hand, its value is by

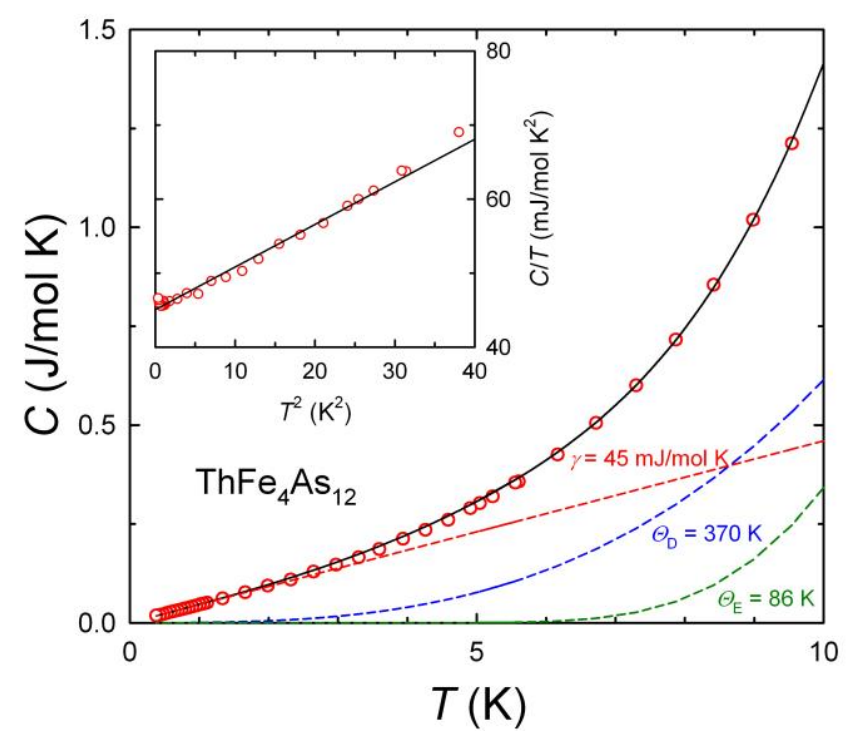

a factor of nearly 2 smaller than the experimentally determined value $\gamma=78 \mathrm{~mJ} / \mathrm{mol}-\mathrm{K}^{2}$ for the itinerant ferromagnet $\mathrm{LaFe}_{4} \mathrm{As}_{12}$ [20]. However, for the latter system, the coefficient $\gamma$ can be substantially enhanced by spin fluctuations.

Fig. 3. Low-temperature specific heat $C$ vs. temperature $T$ of $\mathrm{ThFe}_{4} \mathrm{As}_{12}$. The dashed lines represent contributions of individual terms of the fit (solid line) as described in the text. Inset: Temperature dependence of the specific heat, plotted as $C / T$ vs. $T^{2}$.

Fig. 4 shows the temperature dependence of the electrical resistivity normalized to room-temperature $\rho / \rho_{300 \mathrm{~K}}$ for four $\mathrm{ThFe}_{4} \mathrm{As}_{12}$ single-crystalline samples labelled/oriented as follows: sample \#1/undefined, \#2/[522], $\# 3 /[100] \# 4 /[320]$. The average of the room temperature (RT) values of the resistivity equals $390 \pm 30 \mu \Omega \mathrm{cm}(60$ 
$\mu \Omega \mathrm{cm}$ is the extreme difference of the RT resistivity) while the values of the residual resistivity ratio (RRR) $=\rho(300$ $\mathrm{K}) / \rho(2 \mathrm{~K})$ range from 2.68 to 2.72 .

The figure shows differences in the $\rho(T)$ behavior between different samples. With increasing temperature, $\rho(T)$

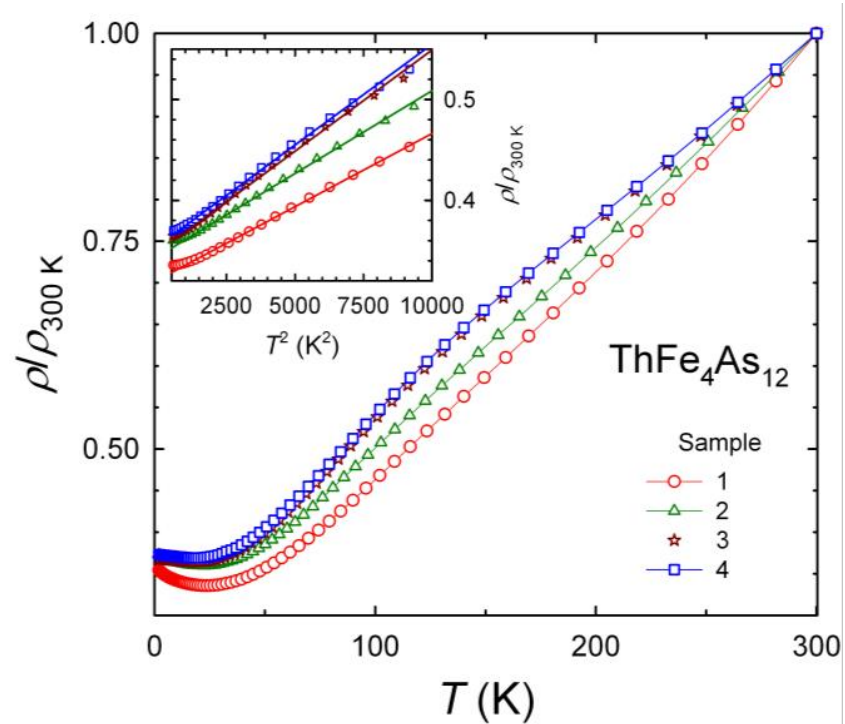
displays a sample dependent shallow minimum at a temperature $T_{\min }$ located between 20 and $25 \mathrm{~K}$.

Fig. 4. Electrical resistivity normalized to the roomtemperature value, $\rho / \rho_{300 \mathrm{~K}}$, vs. temperature $T$ data for four single crystalline $\mathrm{ThFe}_{4} \mathrm{As}_{12}$ samples. Their RRR ranges from $2.68(\# 4)$ to 2.72 (\#1), respectively. Inset: The $\rho / \rho_{300}$ K ratios vs. $T^{2}$ for samples \#1 (the lowest curve), \#2, \#3, and \#4, displaced respectively to higher positions in the figure for clarity.

A quadratic $T$ dependent resistivity $\rho(T)=\rho_{0}+\mathrm{A} T^{2}$ is observed for $30 \mathrm{~K}<T<80 \mathrm{~K}$ and is shown in the inset. The values of the coefficient $A$, in units of $10^{-3} \times \mu \Omega \mathrm{cm} / \mathrm{K}^{2}$, are 5.66, 6.41, 7.83, and 7.80 for samples \#1, \#2, \#3, and \#4, respectively. The slope $\mathrm{d} \rho / \mathrm{d} T$ exhibits a maximum at a temperature $T_{\mathrm{sf}} \sim 90 \mathrm{~K}$, above which the $\rho(T)$ curves bend over slightly and become linear. The $\rho(T)$ curve has a shoulder like contribution that will be discussed latter.

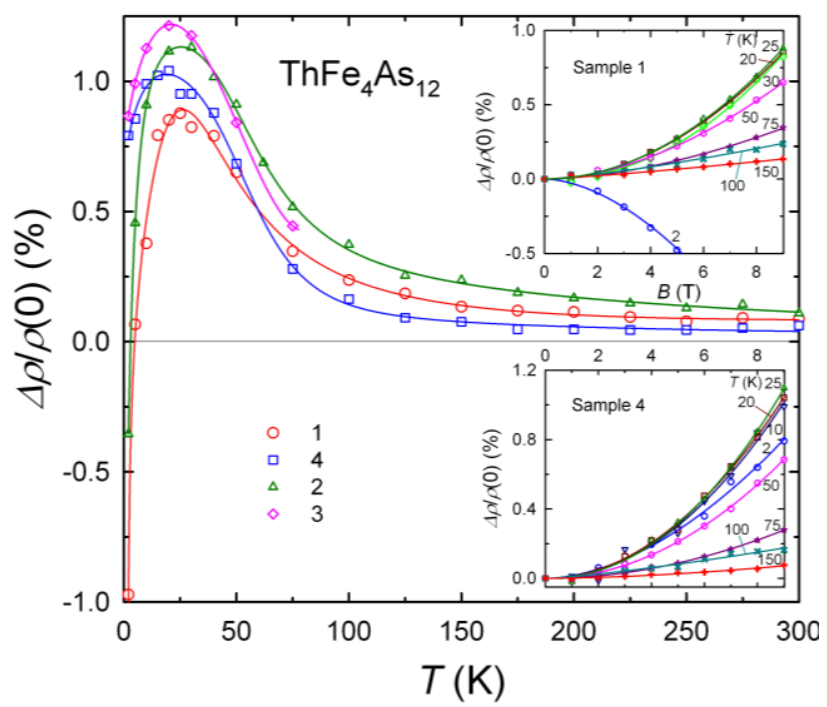

Fig. 5. Non-hysteretic transverse magnetoresistivity, $(M R), \Delta \rho / \rho(0)=[\rho(H)-\rho(0)] / \rho(0)$, vs. temperature $T$ for four selected $\mathrm{ThFe}_{4} \mathrm{As}_{12}$ crystals in a magnetic field of 9 T. Upper inset: $\Delta \rho / \rho(0)$ vs. magnetic field $B$ for crystal \#1 at selected temperatures. Lower inset: $\Delta \rho / \rho(0)$ vs. $B$ for crystal \#4 at selected temperatures.

The effect of a transverse magnetic field on the resistivity of four different $\mathrm{ThFe}_{4} \mathrm{As}_{12}$ single crystals is shown in Fig. 5. The main figure presents the temperature dependence of the magnetoresistivity $(M R), \Delta \rho / \rho(0)=$ $[\rho(H)-\rho(0)] / \rho(0)$, measured in a magnetic field $B=9 \mathrm{~T}$. The $M R$ for crystals \#1 and \#2 show a negative $M R$ at $2 \mathrm{~K}$ 
of the order -0.36 and $-1 \%$, respectively. With increasing temperature, their $M R(T)$ rapidly increases above $+0.8 \%$ at 10 and $15 \mathrm{~K}$, respectively. The samples \#3 and \#4 exhibit such a $M R$ already at $2 \mathrm{~K}$. Then the $M R$ 's pass through sample dependent maxima between 20 and $25 \mathrm{~K}$, rapidly decrease and tend to level off above $100 \mathrm{~K}$ at a positive $M R$ < about $0.1 \%$. When the $M R$ exceeds $+0.8 \%$ below the maxima, the $M R$ 's exhibit a roughly quadratic dependence on magnetic field. Such behavior of the $M R$ persists up to $\sim 75 \mathrm{~K}$, while above $\sim 100 \mathrm{~K}$, the exponent $n$ in the $M R \sim B^{\mathrm{n}}$ dependence decreases to values of $\sim 1.3$. This is illustrated for samples \#1 and \#4 in upper and lower insets in Fig. 5, respectively.

The temperature dependence of the Hall coefficient $R_{\mathrm{H}}(T)$ of samples \#2 and \#4 is shown in Fig. 6 for $2 \leq T \leq 300 \mathrm{~K}$, measured in magnetic fields $1 \mathrm{~T} \leq B \leq 9 \mathrm{~T}$. The inset displays the effect of magnetic field on $R_{\mathrm{H}}$ for $1 \mathrm{~T} \leq B \leq 9 \mathrm{~T}$ at temperatures of 25 and $250 \mathrm{~K}$. This behavior is qualitatively similar to that earlier observed for the semi-metallic compound LuAs [38] except that in the latter, $R_{\mathrm{H}}$ is shifted to lower value and changes sign at $169 \mathrm{~K}$. Quantitatively, $R_{\mathrm{H}}(T, B=1 \mathrm{~T})$ for sample \#4 has a nearly temperature independent value of $R_{\mathrm{H}}=0.0123 \mathrm{~cm}^{3} / \mathrm{C}$ for temperatures $2 \mathrm{~K}$ $\leq T \leq 25 \mathrm{~K}$. Above $\mathrm{T} \sim 25 \mathrm{~K}, R_{\mathrm{H}}$ decreases with $T$ and can be described empirically by the "guide to the eye" curve $R_{\mathrm{H}}=\mathrm{A}+\mathrm{B} / T$ (solid lines) with $\mathrm{A}=2.32 \times 10^{-3} \mathrm{~cm}^{3} / \mathrm{C}$ and $\mathrm{B}=0.379 \mathrm{~cm}^{3} / \mathrm{C} \mathrm{K}$ for sample \#4.

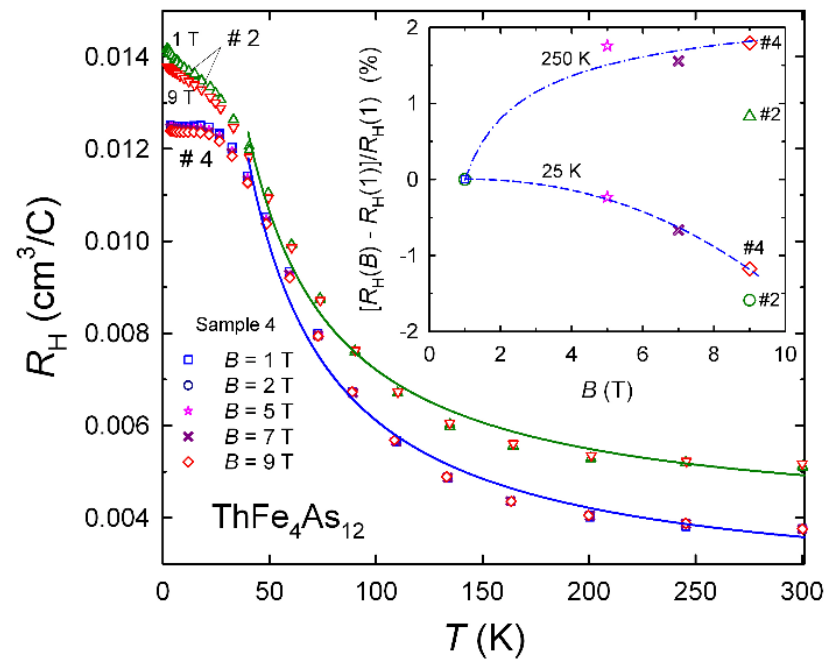

Fig. 6. The Hall coefficient $R_{\mathrm{H}}$ vs. temperature $T$ of samples \#2 and \#4 for $2 \mathrm{~K} \leq T \leq 300 \mathrm{~K}$ in magnetic fields $B$ of $1,2,5,7$, and $9 \mathrm{~T}$, as denoted in the figure. Inset: The Hall coefficient $R_{\mathrm{H}}$ vs $B$ for samples \#2 and $\# 4$ for $1 \mathrm{~T} \leq B \leq 9 \mathrm{~T}$ at temperatures of 25 and $250 \mathrm{~K}$.

The Hall coefficient $R_{\mathrm{H}}(T, B=1)$ for sample \#2 is shifted upward with respect to sample \#4 and decreases with temperature from the value $R_{\mathrm{H}}(T=2 \mathrm{~K}, B=1 \mathrm{~T})=$ $0.0142 \mathrm{~cm}^{3} / \mathrm{C}$ to values roughly described by the "guide to the eye" curve (solid line) above $\sim 40 \mathrm{~K}$ with the

constants $\mathrm{A}=3.78 \times 10^{-3} \mathrm{~cm}^{3} / \mathrm{C}$ and $\mathrm{B}=0.343 \mathrm{~cm}^{3} / \mathrm{C} \mathrm{K}$. It was shown previously [38] that in semimetals, the Hall coefficient at constant $T$ decreases with magnetic field when electrons $n$ are majority carriers and increases with magnetic field when holes $p$ as majority carriers. We may expect a balance between charge carriers, i.e., $n=p$, at temperatures $T_{\text {bal }}$ at which we observe a transition between these two ranges. It was found that $T_{\text {bal }}$ is equal about 84 $\mathrm{K}$ and $36 \mathrm{~K}$ for samples \#2 and \#4, respectively. The inset in Fig. 6 shows $R_{\mathrm{H}}$ vs. $B$ for samples \#2 and \#4 at temperatures 25 and $250 \mathrm{~K}$.

The temperature dependence of the thermoelectric power $S(T)$ is shown in Fig. 7 for $0.4 \mathrm{~K} \leq T \leq 300 \mathrm{~K}$ for four samples of $\mathrm{ThFe}_{4} \mathrm{As}_{12}$, where $S(300 \mathrm{~K})$ varies between $\sim 17.7$ and $31.0 \mu \mathrm{V} / \mathrm{K}$. With increasing temperature, $S(T)$ for samples \#2 and \#4 initially tends towards positive and negative values with rates of 0.06 and $-0.10 \mu \mathrm{V} / \mathrm{K}^{2}$, 
respectively. Thereafter, $S(T)$ for sample \#2 changes sign at $\sim 2 \mathrm{~K}$, and the $S(T)$ data for the both samples go to negative values with an increasing rate until they cross each other and attain minima at $53 \mathrm{~K}, S=-15.2 \mu \mathrm{V} / \mathrm{K}$ and 38 $\mathrm{K}, S=-12.2 \mu \mathrm{V} / \mathrm{K}$ for samples \#2 and \#4, respectively. After turning upward, $S(T)$ reaches -4.4 and $+3.0 \mu \mathrm{V} / \mathrm{K}$ at $170 \mathrm{~K}$ and then increases in rate and attains values of 23.5 and $31.0 \mu \mathrm{V} / \mathrm{K}$ at RT for samples \#2 and \#4, respectively. The behavior of $S(T)$ below $8 \mathrm{~K}$ is shown in the inset of Fig 7 for samples \#2 and \#4. The resistivity and other

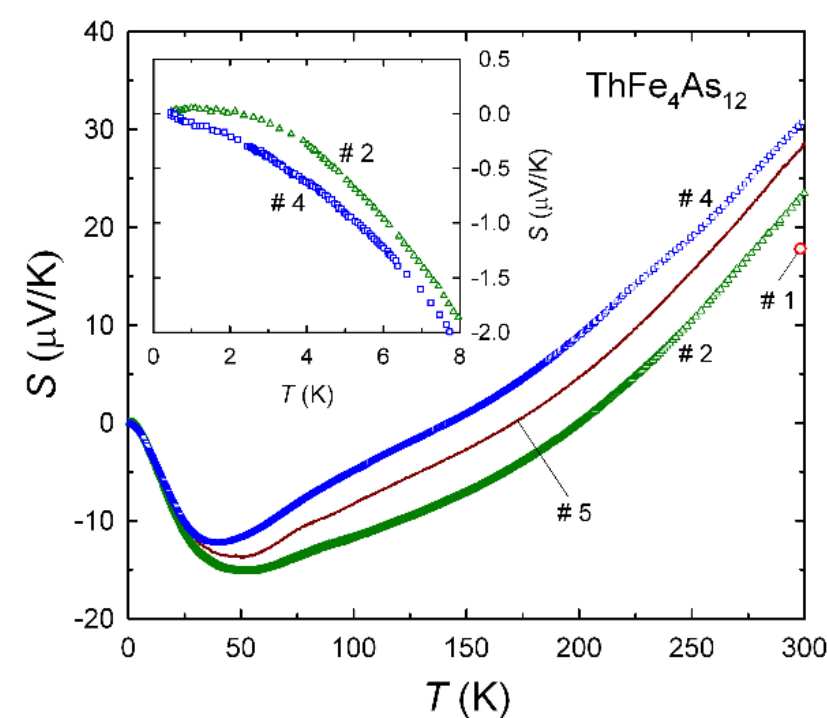

properties, beside $S(T)$, of the sample \#5 were not investigated due to its small dimensions.

Fig. 7. Thermoelectric power $S$ vs. temperature $T$ for $0.3 \mathrm{~K} \leq T \leq 300 \mathrm{~K}$ for four crystals of $\mathrm{ThFe}_{4} \mathrm{As}_{12}$. The inset shows $S(T)$ for $0.3 \mathrm{~K} \leq T \leq 8 \mathrm{~K}$ for crystals \#2 and \# 4 (enlarged scale).

A value of $S / T$ of $+0.062 \mu \mathrm{V} / \mathrm{K}^{2}$ was obtained for sample \#2 by using a linear fit of the data over the temperature range $0.6 \mathrm{~K} \leq T \leq 1.1 \mathrm{~K}$. This procedure yielded a value for $S / T$ of $-0.097 \mu \mathrm{V} / \mathrm{K}^{2}$ for sample \#4

over the temperature range $0.7 \mathrm{~K} \leq T \leq 1.8 \mathrm{~K}$.

\section{Discussion}

The magnetic susceptibility of $\mathrm{ThFe}_{4} \mathrm{As}_{12}$ shows Curie-Weiss behavior $\left(\mu_{\mathrm{eff}}=1.05 \mu_{\mathrm{B}} /\right.$ f.u. $)$ as expected for a nearly ferromagnetic system in which spin fluctuations $\left(\mathrm{SF}_{\mathrm{S}}\right)$ dominate the magnetic susceptibility [39]. In addition, the homologous compound, $\mathrm{LaFe}_{4} \mathrm{As}_{12}$ [20], is an itinerant ferromagnet below $5.2 \mathrm{~K}$ with enhanced spin fluctuations in the paramagnetic state. This was shown by measurements of ${ }^{139} \mathrm{La} \mathrm{NMR},{ }^{75} \mathrm{As} \mathrm{NQR}$, and $\chi(T)$, where the latter conforms to a Curie-Weiss law with $\mu_{\mathrm{eff}}=1.92 \mu_{\mathrm{B}}$ [21]. It is suggestive that the spin fluctuations will contribute a term $\rho_{\mathrm{sf}}(T)=\mathrm{A} T^{2}$ to the total electrical resistivity. Here, the coefficient $\mathrm{A}$ is enhanced below the spin fluctuation temperature $T_{\mathrm{sf}}$ where $\mathrm{d} \rho / \mathrm{d} T$ exhibits a maximum. Above $T_{\mathrm{sf}}, \rho_{\mathrm{sf}}(T)$ bends over and tends towards saturation. This behavior, together with a shoulder-like feature in $\rho(T)$, was anticipated for weakly and nearly ferromagnetic metals $[40,41]$. In fact, these types of characteristics in the resistivity were observed in $\mathrm{MFe}_{4} \mathrm{Sb}_{12}(\mathrm{M}=\mathrm{Ca}, \mathrm{Sr}, \mathrm{Ba})$ skutterudites [42, 43] and $\mathrm{RCo}_{2}(\mathrm{R}=\mathrm{Y}, \mathrm{Lu}, \mathrm{Sc})$ Laves phase compounds [44], which are nearly magnetic metals. We note an increasing tendency for the formation of the shoulder-like contribution to the $\rho(T) / \rho(300)$ vs. $T$ data for $\mathrm{ThFe}_{4} \mathrm{As}_{12}$ when one successively moves from sample \# 1 to \# 4 (Fig. 4). A qualitatively similar, but quantitatively much stronger, variation of $[\rho(T)-\rho(0)] / \rho(300)$ vs. $T$ was observed when moving from $\mathrm{LaFe}_{4} \mathrm{Sb}_{12}$ to $\mathrm{LaFe}_{4} \mathrm{P}_{12}$ 
through $\mathrm{LaFe}_{4} \mathrm{As}_{12}$ [20]. But even in this series of compounds, a saturation of this dependence is weaker than that for $\mathrm{MFe}_{4} \mathrm{Sb}_{12}(\mathrm{M}=\mathrm{Ca}, \mathrm{Sr}, \mathrm{Ba})[42,43]$ or $\mathrm{RCo}_{2}(\mathrm{R}=\mathrm{Y}, \mathrm{Lu}, \mathrm{Sc})$ [44]. The ratio $A / \gamma_{\mathrm{Fe}}^{2}$ (here $\gamma_{\mathrm{Fe}}$ is $\gamma$ per mol $\left.\mathrm{Fe}\right)$ for $\mathrm{ThFe}_{4} \mathrm{As}_{12}$ is between $4.7 \pm 0.4 \times 10^{-5}$ and $6.4 \pm 0.5 \times 10^{-5}\left(\mu \Omega \mathrm{cm} / \mathrm{K}^{2}\right) /\left(\mathrm{mJ} / \mathrm{mol}_{\mathrm{Fe}} \mathrm{K}^{2}\right)^{2}$ and larger than $1 \times 10^{-5}$ $\left(\mu \Omega \mathrm{cm} / \mathrm{K}^{2}\right) /\left(\mathrm{mJ} / \mathrm{mol} \mathrm{K}^{2}\right)^{2}$, the Kadowaki-Woods (KW) [45] value, commonly observed for heavy fermion and some "semi-heavy" fermion compounds $(\sim 50<\gamma<300)$, including compounds classified as SF systems. The compound $\mathrm{ThFe}_{4} \mathrm{As}_{12}$ with $\gamma_{\mathrm{Fe}}=11.2 \mathrm{~mJ} / \mathrm{mol}_{\mathrm{Fe}} \mathrm{K}^{2}$ does not seem to belong to the class of SF systems. In addition, the specific heat coefficients of 78, 57, and 45 for $\mathrm{LaFe}_{4} \mathrm{As}_{12}, \mathrm{LaFe}_{4} \mathrm{P}_{2}[20,46]$, and $\mathrm{ThFe}_{4} \mathrm{As}_{12}$, respectively, seem to be consistent with the trends observed in these systems: itinerant-electron weak ferromagnetism, paramagnetism, and ordinary paramagnetism, respectively. In light of these comments, it seems appropriate to treat our $\mathrm{ThFe}_{4} \mathrm{As}_{12}$ crystals as ordinary paramagnets.

As-based filled skutterudites, grown by the Cd-As flux method, typically have high RRR's; e.g., 75 ( $\left.\mathrm{PrOs}_{4} \mathrm{As}_{12}, \mathrm{PrFe}_{4} \mathrm{As}_{12}, \mathrm{LaFe}_{4} \mathrm{As}_{12}\right), 94\left(\mathrm{PrRu}_{4} \mathrm{As}_{12}\right)$ or $198\left(\mathrm{LaRu}_{4} \mathrm{As}_{12}\right)$ and are definitely regarded as metals [47]. On the other hand, the RRR's for $\mathrm{ThFe}_{4} \mathrm{As}_{12}$, grown by the same method, are only $\sim 2.7$. A single crystal of $\mathrm{ThFe}_{4} \mathrm{P}_{12}$, grown from a $\mathrm{Sn}$ flux, also had a RRR as low as 1.8 (estimated from figure in ref. [25]). Such low RRR's may be observed if stoichiometic semimetals, like rare earth monopnictides, grow with off-stoichiometry defects (see reference [38]) or if a semiconductor, like $\mathrm{CeFe}_{4} \mathrm{As}_{12}$, grown with high level of off-stoichiometry defects, show semimetallic behavior [10]. In those cases, different crystals even grown in the same batch show different electron transport features. One of these is the maximum in $M R(T)$ and the corresponding minimum of $\rho(T)$, observed at low temperatures and related to carrier mobility, which will be discussed later.

The transport properties of $\mathrm{ThFe}_{4} \mathrm{As}_{12}$, determined here, will be analyzed in terms of a two-band model. It consists of a conduction band containing $n$ electrons with mobility $\mu_{\mathrm{e}}$ and a valence band containing $p$ holes with mobility $\mu_{\mathrm{h}}$. In such a case, the Hall coefficient in low magnetic fields is given by [48],

$$
{ }^{0} R_{\mathrm{H}}=\frac{\left(p \mu_{\mathrm{h}}^{2}-n \mu_{\mathrm{e}}^{2}\right)}{|e|\left(n \mu_{\mathrm{e}}+p \mu_{\mathrm{h}}\right)^{2}}
$$

For high magnetic fields, we obtain,

$$
{ }^{B} R_{\mathrm{H}}=\frac{\rho(B)\left[p \mu_{\mathrm{h}}^{2}-n \mu_{\mathrm{e}}^{2}+(p-n) \mu_{\mathrm{e}}^{2} \mu_{\mathrm{h}}^{2} B^{2}\right]}{\left[n \mu_{\mathrm{e}}+p \mu_{\mathrm{h}}+\left(n \mu_{\mathrm{h}}+p \mu_{\mathrm{e}}\right) \mu_{\mathrm{e}} \mu_{\mathrm{h}} B^{2}\right]}
$$

The last term in the numerator of Eq. (4) simply indicates that the change of sign of the slope of ${ }^{B} R_{\mathrm{H}}(B)_{T=\mathrm{const}}$ (see inset, Fig. 6) from negative for $T<T_{\text {bal }}$ to positive for $T>T_{\text {bal }}$, is due to a crossover of holes from being the minority carriers below $T_{\text {bal, }}$, to being the majority carriers above $T_{\text {bal }}$ [38]. Despite this, the Hall coefficient is positive in the entire temperature range because of the clearly higher mobility of hole carriers than that of electron carriers. To 
begin with, the transport coefficients are analyzed in the whole temperature range with the constraint $p-n=0$ (Eq. (3), (5), and (6)), because with increasing temperature above $T_{\text {bal }}$, a decrease of $M R$ and $R_{\mathrm{H}}$ degrades (reduces) the accuracy of the measurements; in particular, $\left|{ }^{\mathrm{B}} R_{\mathrm{H}}-{ }^{0} R_{\mathrm{H}}\right| /{ }^{0} R_{\mathrm{H}} \leq 1.5 \%$. Secondly, the coefficients will be analyzed without the constraint for $T \leq T_{\text {bal }}$ (Eqs. (3), (4), (5), and (6)).

$$
\begin{gathered}
\mu_{\mathrm{e}} \mu_{\mathrm{h}}=\frac{\Delta \rho}{\rho_{0} B^{2}} \\
n \mu_{\mathrm{e}}+p \mu_{\mathrm{h}}=\frac{1}{\rho_{0}|e|}
\end{gathered}
$$

The carrier densities and their mobilities for sample \#2 resulting from the first analysis are presented in Fig. 8. For $\mathrm{ThFe}_{4} \mathrm{As}_{12}$, the mobility first increases with temperature and then passes through a peak of $\mu_{\mathrm{h}}(10 \mathrm{~K})\left[\mu_{\mathrm{e}}(20 \mathrm{~K})\right]$. The concentration of carriers is lowest at $20 \mathrm{~K}$ and equal to $1.8 \times 10^{20} \mathrm{e}^{ \pm} / \mathrm{cm}^{3}$. It increases with temperature, attains a maximum value of $n=p=2.55 \times 10^{20} \mathrm{e}^{ \pm} / \mathrm{cm}^{3}$ at $125 \mathrm{~K}$, and then decreases roughly linearly with temperature to a value of $2.25 \times 10^{20} \mathrm{e}^{ \pm} / \mathrm{cm}^{-3}$ at $300 \mathrm{~K}$. The mobility of the carriers behaves qualitatively similar to that observed earlier for the LuAs semimetal [38].

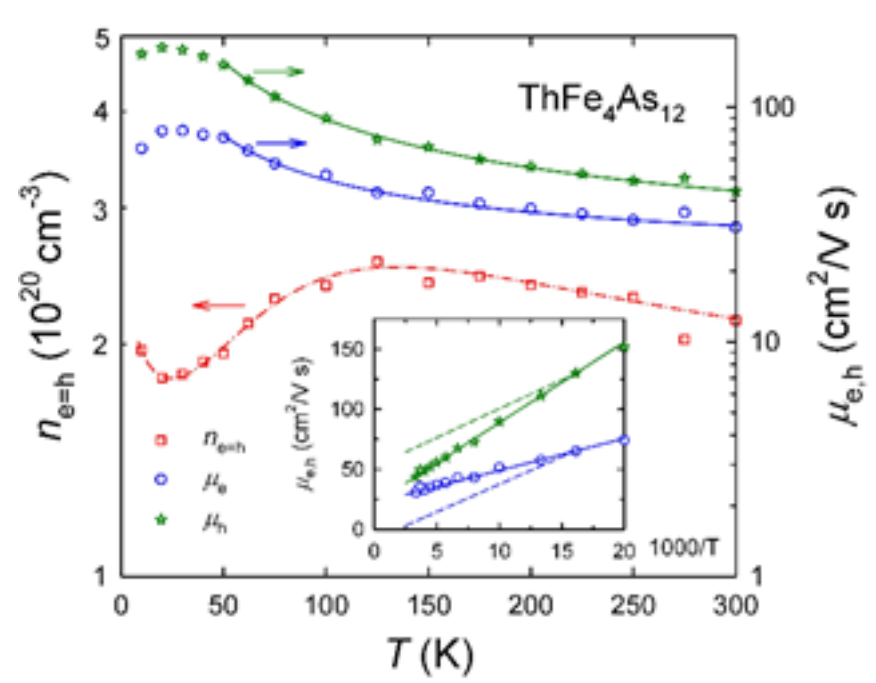

Fig. 8. Equal conduction electron and hole concentrations (lower curve) and mobility (upper curves) vs. temperature for sample \#2. The inset shows the mobility vs. reciprocal temperature $1000 / T$ for $60<T \leq 300 \mathrm{~K}$. Solid points represent experimental data for the hole $\left(\mu_{\mathrm{h}}\right)$ and electron $\left(\mu_{\mathrm{e}}\right)$ mobility approximated by the solid lines. Upper and lower broken lines represent the hole and electron mobilities that correspond to effective masses $\left(m_{\mathrm{h}} * / m_{0}\right)=0.37$ and $\left(m_{\mathrm{e}} * / m_{0}\right)=2.01$ to be consistent with the behavior of the thermoelectric power for sample \#2 vs. temperature.

For $\mathrm{ThFe}_{4} \mathrm{As}_{12}$, the mobilities first increase with temperature and then pass through a peak of $\mu_{\mathrm{h}}(10 \mathrm{~K})\left[\mu_{\mathrm{e}}(20 \mathrm{~K})\right]$, equal to $179.5[79.4] \mathrm{cm}^{2} / \mathrm{V} \cdot \mathrm{s}$. Upon further increase of temperature, the mobility of both electrons and holes decreases roughly as $\sim 1000 / T$ for $62 \leq T \leq 300 \mathrm{~K}$ (see inset in Fig. 8) and attains the value of $\mu_{\mathrm{h}}\left(\mu_{\mathrm{e}}\right.$ ), equal to 43.6 (31) $\mathrm{cm}^{2} / \mathrm{V} \cdot \mathrm{s}$ at $300 \mathrm{~K}$. A peak in the carrier mobility is a characteristic feature for a semiconductor (also observed in semimetallic LuAs) when two mechanisms simultaneously control carrier scattering; e.g., by charged impurity $\mu_{\mathrm{I}}(\sim T$ $3 / 2$ ) and by acoustic lattice $\mu_{\mathrm{L}}\left(\sim T^{-3 / 2}\right)$ scattering. A change of the exponent from $-3 / 2$ to -1 is observed in simple metals because most of the scattering events involve carriers near the Fermi energy [49]. 
According to Behnia et al. [50], the thermoelectric power should obey the relation $q=\left(N_{\mathrm{A}} \times e / \gamma\right)(S / T)$ in the zerotemperature limit. Here, $\gamma$ is the electronic specific heat coefficient, $N_{\mathrm{A}}$ is Avogadro's number, $e$ is the electron charge, and the product $N_{\mathrm{A}} \times e=9.65 \times 10^{4} \mathrm{C} / \mathrm{mol}$ is the Faraday number. The dimensionless quantity $q$ corresponds to the density of carriers per formula unit for the free electron gas model with an energy-independent relaxation time. (The relation for $q$ was more rigorously derived by Zlatić et al. [51]). Using the determined $S / T$ values as well as $\gamma$, the values $q=0.13$ and $q=-0.22$ were calculated for samples \#2 and \#4, respectively; their Hall carrier densities, determined in a one-band model (HCDOBM) at $4 \mathrm{~K}$, are equal to $4.5 \times 10^{20}$ and $5.1 \times 10^{20} e^{+} / \mathrm{cm}^{3}$, respectively. These values can be compared with $q=36,13$, and -9 for $\mathrm{CeFe}_{4} \mathrm{As}_{12}$ crystals, where the HCDOBM values at $4 \mathrm{~K}$ were $1,0 \times 10^{20}, 1.5 \times 10^{20}$, and $3.5 \times 10^{20} e^{+} / \mathrm{cm}^{3}$, respectively [10]. The $\mathrm{CeFe}_{4} \mathrm{As}_{12}$ crystals reveal phenomena associated with $f$-electron - conduction electron hybridization and heavily doped semiconducting solids. It is worth noticing that for Kondo insulators like $\mathrm{CeOs}_{4} \mathrm{As}_{12}$ [11] and CeNiSn [49], $q=89$ and 107, respectively. On the other hand, Behnia et al. [50] found $0.5 \leq|q| \leq 2$ for a wide range of different metals. The $q$ values determined for $\mathrm{ThFe}_{4} \mathrm{As}_{12}$ are slightly lower in this range and are clearly lower than $q$-values for compounds whose semiconducting phenomena are associated with $f$-electron - conduction electron hybridization.

In the case of the thermoelectric power $S(T)$ of a two-band conductor, each band contributes to the total $S(T)$ in proportion to its contribution to the total electrical conductivity of the conductor. Thus, the total $S(T)$ consists of the thermoelectric power $S_{\mathrm{e}}(T)$ and electrical conductivity $\sigma_{\mathrm{e}}(T)=|e| n \mu_{\mathrm{e}}$ of the electron conduction band and the corresponding $S_{\mathrm{h}}(T)$ and $\sigma_{\mathrm{h}}(T)=|e| p \mu_{\mathrm{h}}$ of the hole band. Both contributions enter the $S(T)$ formula as follows [52]:

$$
\begin{aligned}
& S(T)=S_{\mathrm{e}, \mathrm{c}}+S_{\mathrm{h}, \mathrm{c}}=\frac{S_{\mathrm{e}}(T) \sigma_{\mathrm{e}}(T)}{\sigma_{0}(T)}+\frac{S_{\mathrm{h}}(T) \sigma_{\mathrm{h}}(T)}{\sigma_{0}(T)}=\frac{S_{\mathrm{e}}(T) n \mu_{\mathrm{e}}+S_{\mathrm{h}}(T) p \mu_{\mathrm{h}}}{\left(n \mu_{\mathrm{e}}+p \mu_{\mathrm{h}}\right)} \\
& S_{\mathrm{e}, \mathrm{h}}(T)=x \frac{\left(\pi^{2} k_{\mathrm{B}}^{2} T\right)}{3 q E_{\mathrm{F}(\mathrm{e}, \mathrm{h})}} ; \quad S_{\mathrm{e}}(T)=-x z\left(\frac{m_{\mathrm{e}}^{*} T}{m_{0} n^{2 / 3}}\right) ; \quad S_{\mathrm{h}}(T)=+x z\left(\frac{m_{\mathrm{h}}^{*} T}{m_{0} p^{2 / 3}}\right)
\end{aligned}
$$

where $q, \sigma_{0}$ and $m_{0}$ are the carrier charge, total electrical conductivity, and free electron mass, respectively, while $z=\frac{2 m_{0} k_{\mathrm{B}}^{2} \pi^{2 / 3}}{e \hbar^{2} 3^{5 / 3}}$. The Fermi energies $E_{\mathrm{Fe}}$ and $E_{\mathrm{Fh}}$ are defined as an energy difference between the Fermi level and the bottom edge of the conduction band or the top edge of the valence band and the Fermi level, respectively. Temperature independent $x$ parameters between 2 and 3 [53] were observed for various simple metals at high temperatures. We have assumed $x=3$ and fit the calculated $S(T)$ to the experimental data of sample \#2 (Fig. 9) as described below.

The high magnetic field Hall coefficients are only slightly larger than the low field ones for $62 \leq T \leq 300 \mathrm{~K}$. However, the differences were large enough to identify holes as the majority carriers, but inadequate to determine the 
exact difference between the hole and electron carrier densities. In order to fit the calculated $S(T)$ - using the estimated $n=p, \mu_{\mathrm{h}}$ and $\mu_{\mathrm{e}}$ data for sample \#2 - to the experimental $S(T)$ data, we have to account for a higher contribution of the majority carriers (holes, above $\sim 84 \mathrm{~K}$ ) to the electrical conductivity. Both carrier density and mobility enter linearly into the conductivity. However, the temperature dependence of the mobility can be more easily modified than the density, especially in the $62 \mathrm{~K} \leq T \leq 300 \mathrm{~K}$ range. Then, the mobility can be approximated by $\mu_{\mathrm{h}}=21.64+6709 / T$ for holes and $\mu_{\mathrm{e}}=22.36+2663 / T$ for electrons. Unfortunately, in contrast to the LuAs case [38], where both mobilities - holes and electrons - are nearly in parallel with decreasing $1000 / T$, for $\mathrm{ThFe}_{4} \mathrm{As}_{12}$, they do not change in a similar way.

For the thermoelectric power calculations, we use the following modified mobility temperature dependences: A; $\left(\mu_{\mathrm{h}}=32.36+6035 / T, \mu_{\mathrm{e}}=11.64+3339 / T\right), \mathrm{B} ;\left(\mu_{\mathrm{h}}=41.97+5438 / T, \mu_{\mathrm{e}}=2.03+3935 / T\right)$ and $\mathrm{C}$; $\left(\mu_{\mathrm{h}}=52.30+4798 / T, \mu_{\mathrm{e}}=-8.30+4575 / T\right)$ which are valid for $T \geq 62 \mathrm{~K}$. Below this temperature, $\mu_{\mathrm{h}}(T)$ and $\mu_{\mathrm{e}}(T)$ were taken as calculated from Eqs. (4) - (6) (original ones). The terms $\left(\mu_{\mathrm{h}}+\mu_{\mathrm{e}}\right)$ for the modified mobility are held constant for given temperature and therefore the temperature dependence of the resistivity remains the same as the experimental one. The calculated thermoelectric power data are plotted in Fig. 9 by stars - A, squares - B, and circles - C. The effective masses $m_{\mathrm{h}}{ }^{*} / m_{0}$ and $m_{\mathrm{e}}{ }^{*} / m_{0}$ have been obtained from fits of Eq. (5) to $S(62 \mathrm{~K})$ and $S(250 \mathrm{~K})$, and they are (5.52 and 12.24), (1.014 and 3.298), and (0.366 and 2.011) for the A, B and C models, respectively.

The calculated $S(T)$ curves describe reasonably well the experimental $S(T)$ data for sample \#2. The similarity between the calculated and measured $S(T)$ curves in the range $62 \mathrm{~K} \leq T \leq 300 \mathrm{~K}$ improves slightly when the effective masses in the fits are decreased, where the mobility was corrected to account for the majority of hole carriers in this range. On the other hand, for $T<62 \mathrm{~K}$ where the majority carriers are electrons, we did not apply any correction for the carrier concentrations or mobilities; therefore, the calculated $S(T)$ curves compare less favorably to the experimental curves.

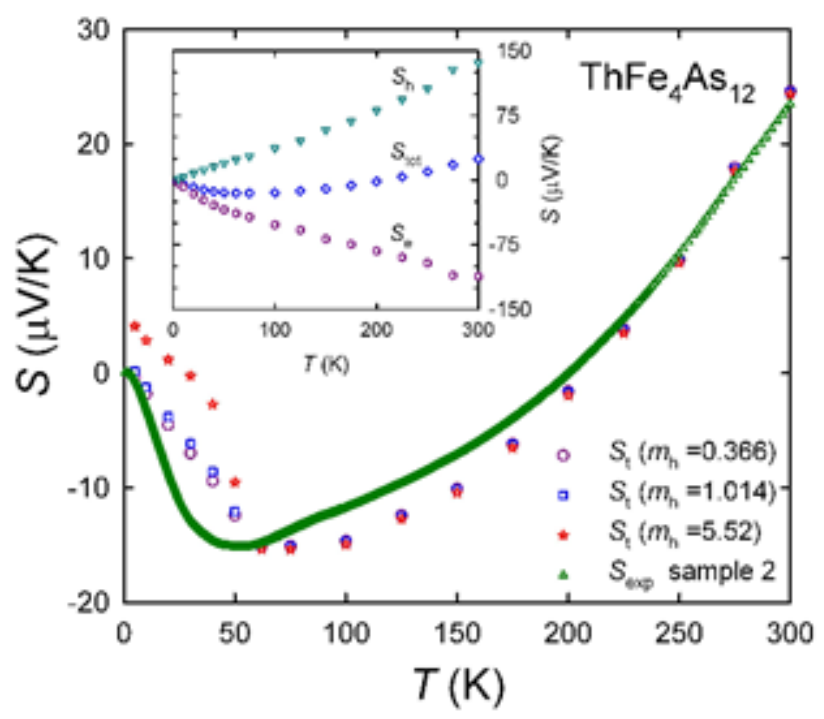

Fig. 9. Thermoelectric power $S$ vs. temperature $T$ for $0.4 \mathrm{~K}<T<300 \mathrm{~K}$ for sample \#2 (solid line). The calculated $S_{\mathrm{c}}(T)$ (circles, squares and stars) of sample \#2 is based on the charge density and mobility of the carriers, as described in the text. Inset: the calculated valence $S_{\mathrm{h}, \mathrm{c}}$ and conduction $S_{\mathrm{e}, \mathrm{c}}$, band contributions to the total $S(T)$ for sample \#2 (see Eq. (7)). 
Analysis of the $\rho(T), M R$, and $R_{\mathrm{H}}(T, B)$ data for crystal \#4, assuming $n=p$, leads to a qualitatively similar temperature dependence of $n(p), \mu_{\mathrm{h}}$, and $\mu_{\mathrm{e}}$ to those presented in Fig. 8 for crystal $\# 2$. The $\mu_{\mathrm{h}}$ and $\mu_{\mathrm{e}}$ pass through a maximum at $T \approx 25 \mathrm{~K}$ and decrease with temperature as $\mu_{\mathrm{h}}=-5.798+6624 / T$ and $\mu_{\mathrm{e}}=-1.034+3384 / T$. Fitting the calculated $S(T)$ (Eq. (7)) to the experimental data, we obtained satisfactory agreement in the range $50<T<175 \mathrm{~K}$ for modified mobility to $\mu_{\mathrm{h}}=11.00+5780 / T$ and $\mu_{\mathrm{e}}=-17.83+4220 / T$ and effective masses $m_{\mathrm{h}}{ }^{*} / m_{0}=0.347$ and $m_{\mathrm{e}}{ }^{*} / m_{0}=1.772\left(m_{\mathrm{e}}{ }^{*} / m_{\mathrm{h}}{ }^{*}=5.1\right)$. This result is close to that found for crystal \#2 for modified mobility C data: $m_{\mathrm{e}}{ }^{*} / m_{\mathrm{h}}{ }^{*}$ $=5.5$.

The carrier concentration calculated with an $n=p$ constraint (Eqs. (3), (5), (6)) as well as without the constraint (Eqs. (3), (4), (5), (6)) at $T_{\text {bal }}$ is $n(p)=2.32 \times 10^{20} \mathrm{e} / \mathrm{cm}^{3}$ for crystal \#2 $\left(T_{\text {bal }}=84 \mathrm{~K}\right)$ and $n(p)=1.75 \times 10^{20}$ $\mathrm{e} / \mathrm{cm}^{3}$ for crystal \#4 ( $\left.T_{\text {bal }}=36 \mathrm{~K}\right)$. The limitation free calculations for $T<0.7 T_{\text {bal }}$ reveal the presence of a charge imbalance in the number of electrons (holes) $n=3.3(7) \times 10^{20}\left(p=1.1(6) \times 10^{20}\right) \mathrm{e} / \mathrm{cm}^{3}$ and $n=1.8(7) \times 10^{20}(p=$ $\left.1.4(7) \times 10^{20}\right) \mathrm{e} / \mathrm{cm}^{3}$ for crystals \#2 and \#4, respectively. Ionization of off-stoichiometric donor defects are presumably the source of electrons responsible for the charge imbalance. However, with the temperature increase above $\sim 0.7 T_{\text {bal }}$, an increase of hole density, mainly at the expense of electron density, is observed. This is presumably due to localization of electrons on immersing the empty states of the defects in the electron sea below $E_{\mathrm{F}}$.

More so in crystal \#2 than in crystal \#4, defects are the reason for the electron density increase in the electron band by $1.50 \times 10^{20} \mathrm{e} / \mathrm{cm}^{3}$ and in the valence band by $0.31 \times 10^{20} \mathrm{e} / \mathrm{cm}^{3}$. This means that the ratio of density of states at the Fermi level, $E_{\mathrm{F}}$, in the electron band to that in the hole band is equal to about 4.8 and is consistent with the ratio of the effective masses of electrons and holes determined for crystal \#2. A similar result is obtained when using the modified carrier mobility; for crystal \#2, it is case $\mathrm{C}\left(m_{\mathrm{h}}{ }^{*} / m_{\mathrm{e}}{ }^{*}=5.5\right)$ and one specified for crystal $\# 4\left(m_{\mathrm{h}}{ }^{*} / m_{\mathrm{e}}{ }^{*}=5.1\right)$. Thus, assuming, for simplicity, $m_{\mathrm{h}}{ }^{*} / m_{0}=0.37, m_{\mathrm{e}}{ }^{*} / m_{0}=2.0$ and parabolic bands, the Fermi energy for crystal \#2 is $E_{\mathrm{F}}$ $=0.088 \mathrm{eV}$ for the electron band, $E_{\mathrm{F}}=0.234 \mathrm{eV}$ for the hole band, and $0.322 \mathrm{eV}$ for the difference between the bottom of electron band and the top of the hole band. Corresponding values for crystal \#4 are equal to 0.060, 0.274 , and $0.334 \mathrm{eV}$. The electronic structure parameters so obtained show that in the observed carrier concentration range their effective masses depend rather weakly on the increase of the carrier concentration. On the other hand, the rate of increase of electron carriers is $\sim 5$ times larger than the rate of decrease of hole carriers and overlap of hole and electron bands (OHEB) remains unchanged in the limit of accuracy.

As noted in the Introduction, we expect semiconducting, or semimetallic properties and charge balance for fully occupied Th crystallographic positions in $\mathrm{ThFe}_{4} \mathrm{As}_{12}$. According to the Zintl concept, (discussed in Section 1), one can expect a narrow semiconducting gap, or even semimetallic properties for the completely filled skutterudite $\mathrm{ThFe}_{4} \mathrm{As}_{12}$.

Treating the measured transport coefficients of the $\mathrm{ThFe}_{4} \mathrm{As}_{12}$ crystals studied with a two carrier band model without the constraint reveals for crystal \#4 an $n-p=0.40 \times 10^{20} \mathrm{e} / \mathrm{cm}^{3}$ charge imbalance for crystal \#4, which is 
equivalent to a $0.29 \%$ excess of fully ionized tetravalent Th atoms which act as electron-doping off-stoichiometric defects (donors). The charge imbalance of crystal \#2 increases to $2.21 \times 10^{20} \mathrm{e} / \mathrm{cm}^{3}$ (i.e., $1.57 \%$ of Th) and consistently, the shift of $S(T)$ for crystal \#2 to more negative values is observed. $S(300 \mathrm{~K})$ is equal to 31.0, 23.5, and $17.7 \mu \mathrm{V} / \mathrm{K}$ for samples \# 4, 2, and 1 (Fig. 7), respectively. The $S(300 \mathrm{~K}), n-p$, and $n$ data for samples \#2 and \#4 were approximated to $n-p=0$ ( hypothetical, defect free sample \# 5) and to $S(300 \mathrm{~K})=17.7 \mu \mathrm{V} / \mathrm{K}$ (sample \# 1) using the $S^{-3 / 2} \sim n$ and $S^{-3 / 2} \sim(n-p)$ relations. Then the $S(300 \mathrm{~K})=33.6 \mu \mathrm{V} / \mathrm{K}$ at $n=p=1.54 \times 10^{20} \mathrm{e} / \mathrm{cm}^{3}$ (charge balance of the carriers), and the OHEB $=0.335 \mathrm{eV}$ data were obtained for sample \# 5. The data given in italic result from the extrapolations. On the other hand the extrapolations done for $S(300 \mathrm{~K})=17.7$ (sample \#1) allowed us to estimate $n=5.71 \times 10^{20} \mathrm{e} / \mathrm{cm}^{3}, p=0.68 \times 10^{20} \mathrm{e} / \mathrm{cm}^{3}$, the imbalance equivalent to $3.6 \%$, an excess of the fully ionized tetravalent Th atoms, and the OHEB equal to $0.270 \mathrm{eV}$. Such a large overlap the hole and electron bands excludes existence of an energy gap in $\mathrm{ThFe}_{4} \mathrm{As}_{12}$ and indicates its semimetallic ground state. It means that the ground state of $\mathrm{ThFe}_{4} \mathrm{As}_{12}$ differs from the semiconducting ground state of the Ce-filled skutterudites presented in the Introduction. Such behavior of the latter $f$-electron compounds is consistent with the hybridization gap model, first proposed by $\mathrm{N}$. F. Mott [54].

Our analysis of experimental data shows indeed the overlap of valence and conduction bands in $\mathrm{ThFe}_{4} \mathrm{As}_{12}$. In contrast to it, the calculated electronic structure for sister compound $\mathrm{ThFe}_{4} \mathrm{P}_{12}[31,32]$ anticipates an energy gap. The calculations show a high density of states at the bottom of the conduction band and a low density of states at the top of the valence band. It is possible that this band features correspond to the heavy electron and light hole bands found in $\mathrm{ThFe}_{4} \mathrm{As}_{12}$. Beside of that the measured resistivity of $\mathrm{ThFe}_{4} \mathrm{P}_{12}$ increases with temperature $[24,29]$ like that of $\mathrm{ThFe}_{4} \mathrm{As}_{12}$ and therefore electronic structure calculations is desired to answer a question whether As for $\mathrm{P}$ atoms replacement could account for closing the energy gap in $\mathrm{ThFe}_{4} \mathrm{As}_{12}$.

\section{Concluding remarks}

The residual resistivity ratio (RRR) of the $\mathrm{ThFe}_{4} \mathrm{As}_{12}$ crystals, grown by the Cd:As flux method, varied between 2.68 and 2.72. Measurements of transport coefficients, i.e., resistivity, magnetoresistivity, Hall coefficient and thermoelectric power, for these samples were performed for temperatures $0.4 \leq T \leq 300 \mathrm{~K}$ and magnetic field $0 \leq$ $B \leq 9 \mathrm{~T}$. The data for the transport coefficients were found to be a sample dependent. They were analysed in terms of a two-band model. The analyses revealed that at low temperature the holes $(p)$ are minority carriers. An increase of temperature causes an increase of the hole density accompanied with a decrease of the density of electron carriers. A charge balance of the carriers is reached at a temperature $T_{\text {bal }}$; for $T>T_{\text {bal }}$, holes become majority carriers.

For one of the $\mathrm{ThFe}_{4} \mathrm{As}_{12}$ crystals examined, at $T_{\text {bal }}=36 \mathrm{~K}$, the charge densities were $n=p=1.75 \times 10^{20}$ $\mathrm{e} / \mathrm{cm}^{3}$ and the mobilities were $\mu_{\mathrm{h}}=155 \mathrm{~cm}^{2} / \mathrm{V} \mathrm{s}$ and $\mu_{\mathrm{e}}=78 \mathrm{~cm}^{2} / \mathrm{V}$ s for holes and electrons, respectively, while for $T$ $<0.7 T_{\text {bal }}$, the charge imbalance was $n-p=0.40 \times 10^{20} \mathrm{e} / \mathrm{cm}^{3}$. For another $\mathrm{ThFe}_{4} \mathrm{As}_{12}$ with $T_{\text {bal }}=84 \mathrm{~K}$, the corresponding values were $n=p=2.32 \times 10^{20} \mathrm{e} / \mathrm{cm}^{3}, \mu_{\mathrm{h}}=102 \mathrm{~cm}^{2} / \mathrm{V} \mathrm{s}$, and $\mu_{\mathrm{e}}=54 \mathrm{~cm}^{2} / \mathrm{V} \mathrm{s}$, and the charge 
imbalance $n-p=2.21 \times 10^{20} \mathrm{e} / \mathrm{cm}^{3}$ for $T<0.7 T_{\text {bal. }}$. The difference of the carrier densities between these two crystals reveals that the density of states in the electron band of $\mathrm{ThFe}_{4} \mathrm{As}_{12}$ at the Fermi level is about 5 times larger than that in the hole band. This estimation is in agreement with the behavior of the thermoelectric power $S(T)$.

The magnetic susceptibility obeys a modified Curie-Weiss law with a negative Curie-Weiss temperature $\Theta$ $=-83.7 \mathrm{~K}$ and yields an effective paramagnetic moment $\mu_{\mathrm{eff}}=1.05 \mu_{\mathrm{B}} / \mathrm{mol}$, presumably due to spin fluctuations of electrons from incompletely filled $3 d$ levels. Consistently, the heat capacity measurement yields an electronic specific heat coefficient $\gamma=45(1) \mathrm{mJ} / \mathrm{mol} \mathrm{K}^{2}$ and an Einstein temperature $\Theta_{\mathrm{E}}=86(1) \mathrm{K}$.

Acknowledgments

The research at UCSD was supported by the U.S. Department of Energy, Office of Basic Energy Sciences, Division of Materials Sciences and Engineering under Grant No. DE-FG02-04ER46105.

The authors would like to thank V. Kinzhybalo for his helpful contribution to the Rietveld structure refinement.

\section{References}

[1] M. B. Maple, E. D. Bauer, N. A. Frederick, P. -C. Ho, W. M. Yuhasz, and V. S. Zapf, Physica B 328 (2003) 2933.

[2] Y. Aoki, H. Sugawara, H. Harima, and H. Sato, J. Phys. Soc. Jpn. 74 (2005) 209-221.

[3] M. B. Maple, Z. Henkie, R. E. Baumbach, T. A. Sayles, N. P. Butch, P.-C. Ho, T. Yanagisawa, W. M. Yuhasz, R. Wawryk, T. Cichorek, and A. Pietraszko, J. Phys. Soc. Jpn. 77 Suppl. A (2008) 7-13.

[4] H. Sato, D. Kikuchi, K. Tanaka, M. Ueda, H. Aoki, T. Ikeno, S. Tatsuoka, K. Kuwahara, Y. Aoki, M. Kohgi, H. Sugawara, K. Iwasa, and H. Harima, J. Phys. Soc. Jpn. 77 Suppl. A (2008) 1-6.

[5] H. Sugawara, S. Osaki, M. Kobayashi, T. Namiki, S. R. Saha, Y. Aoki, and H. Sato, Phys. Rev. B 71 (2005) 125127 1-6.

[6] D. J. Braun and W. Jeitschko, J. Solid State Chem. 32 (1980) 357-363.

[7] D. J. Braun and W. Jeitschko, J. Less-Common Met. 72 (1980) 147-156.

[8] C. Sekine, N. Hoshi, K. Takeda, T. Yoshida, I. Shirotani, K. Matsuchira, M. Wakeshima, and Y. Hinatsu, J. Magn. Magn. Mater. 310 (2007) 260-262.

[9] R. E. Baumbach, P.-C. Ho, T. A. Sayles, M. B. Maple, R. Wawryk, T. Cichorek, A. Pietraszko, and Z. Henkie, J. Phys.: Condens. Matter 20 (2008) 075110 1-7.

[10] R. Wawryk, Z. Henkie, A. Pietraszko, T. Cichorek, L. Kępiński, A. Jezierski, J. Kaczkowski, R. E. Baumbach, and M. B. Maple, Phys. Rev B 84 (2011) 165109 1-11.

[11] R. E. Baumbach, P.-C. Ho, T. A. Sayles, M. B. Maple, R. Wawryk, T. Cichorek, A. Pietraszko, and Z. Henkie, PNAS - Proc. Nat'1. Acad. Sci. USA 105 (2008) 17307-17311.

[12] M. B. Maple and D. K. Wohlleben, AIP Conf. Proc. 18 (1974) 447-462. 
[13] A. Menth, E. Buehler, and T. H. Geballe, Phys. Rev. Lett. 22 (1969) 295-297.

[14] K. W. H. Stevens, J. Phys. C: Solid State Phys., 9 (1976) 1417-1428.

[15] A. C. Hewson, The Kondo Problem to Heavy Fermions (Cambridge Univ. Press, Cambridge 1993, U.K.).

[16] T. Cichorek, Ł. Bochenek, R. Wawryk, and Z. Henkie, Phys. Status Solidi B 250 (2013) 646-649.

[17] H. Sato, H. Sugawara, Y. Aoki, and H. Harima, Magnetic Properties of Filled Skutterudites, in: K. H. J. Buschow (ed.) Handbook of Magnetic Materials Vol. 18, North-Holland is an imprint of Elsevier, Amsterdam, 2009, pp. 1-110 (chapter 1).

[18] C. Sekine, K. Akahira, K. Ito, and T. Yagi, J. Phys. Soc. Jpn.78 (2009) 093707 1-4.

[19] C. Sekine, K. Ito, K. Akahira, Y. Kawamura, Y. Qi Chen, H. Gotou, and K. Matsuhira, J. Phys.: Conf. Series 592 (2015) $012031-6$.

[20] S. Tatsuoka, H. Sato, K. Tanaka, M. Ueda, D. Kikuchi, H. Aoki, T. Ikeno, K. Kuwahara, Y. Aoki, H. Sugawara, and H. Harima, J. Phys. Soc. Jpn. 77 (2008) 033701 1-4.

[21] B. Nowak, O. Żogał, A. Pietraszko, R. E. Baumbach, M. B. Maple, and Z. Henkie, Phys. Rev, B 79 (2009) $2144111-9$.

[22] H. Anno, K. Matsubara, T. Caillat, and J.-P. Fleurial, Phys. Rev. B 62 (2000) 10737-10743.

[23] S. C. Sevov, in Intermetallic Compounds - Principles and Practice Progress. Volume 3 (eds. Westbrook, J. H. \& Freisher, R. L.) 113 - 132 (John Wiley \& Sons, LTD, 2002).

[24] H. Luo, J. W. Krizan, L. Muechler, N. Haldolaarachchige, T. Klimczuk, W. Xie, M. K. Fuccillo, C. Felser, and R. J. Cava, Nature Commun. 6 (2015), 6489 1-10.

[25] S. V. Dordevic, N. R. Dilley, E. D. Bauer, D. N. Basov, M. B. Maple, and L. Degiorgi, Phys. Rev. B 60 (1999) 11321-11328.

[26] G. P. Meisner, M. S. Torikachvili, K. N. Yang, M. B. Maple, and R. P. Guertin, J. Appl. Phys. 57 (1985) $3073-$ 3075.

[27] R. Khenata, A. Bouhemadou, A. H. Reshak, R. Ahmed, B. Bouhafs, D. Rached, Y. Al-Douri, and M. Rérat, Phys. Rev. B 75 (2007) 195131 1-7.

[28] M. Ameri, S. Amel, B. Abidri, I. Ameri, Y. Al-Douri, B. Bouhafs, D. Varshney, A. Aze-Eddine, and L. Nadia, Mater. Sci. Semicond. Process 27 (2014) 368-379.

[29] D. H. Galván, N. R. Dilley, M. B. Maple, A. Posada-Amarillas, A. Reyes-Serrato, and J. C. Samaniego Reyna, Phys. Rev. B 68 (2003) 115110 1-9.

[30] M. S. Torikachvili, J. W. Chen, Y. Dalichaouch, R. P. Guertin, M.W. McElfresh, C. Rossel, M. B. Maple, and G. P. Meisner, Phys. Rev. B 36 (1987) 8660-8664.

[31] K. Takegahara and H. Harima, Physica B 329-333 (2003) 464-466.

[32] Z.-Z. Cheng, B. Xu, and Z. Cheng, Commun. Theor. Phys. (Beijing, China) 49 (2008) 1049-1051.

[33] Z. Henkie, M. B. Maple, A. Pietraszko, R. Wawryk, T. Cichorek, R. E. Baumbach,W. M. Yuhasz, and P.-C. Ho, J. Phys. Soc. Jpn. 77 (2008) Suppl. A 128-134.

[34] W. E. Gardner and J. Penfold, Phil. Mag. 11 (1965) 549-559. 
[35] G. A. Bain and J. F. Berry, J. Chem. Education 85 (2008) 532-536.

[36] K. Matsuhira, C. Sekine, M. Wakeshima, Y. Hinatsu, T. Namiki, K. Takeda, I. Shirotani, H. Sugawara, D. Kikuchi, and H. Sato, J. Phys. Soc. Jpn. 78 (2009) 124601 1-4.

[37] R. Higashinaka, K. Takeda, T. Namiki, Y. Aoki, and H. Sato, J. Phys. Soc. Jpn. 82 (2013) 114710 1-6.

[38] O. Żogał, R. Wawryk, M. Matusiak, and Z. Henkie, J. Alloys Compd. 587 (2014) 190-198.

[39] T. Moriya and K. Ueda, Solid State Commun. 15 (1974) 169-172.

[40] K. Ueda and T. Moriya, J. Phys. Soc. Jpn. 39 (1975) 605-615.

[41] B. Coqblin, J. Iglesias-Sicardi, and R. Jullien, Contemp. Phys. 19 (1978) 327-354.

[42] T. Takabatake, E. Matsuoka, S. Narazu, K. Hayashi, S. Marimoto, T. Sasakawa, K. Umeo, and M. Sera, Physica B 363 (2006) 93-102.

[43] W. Schnelle, A. Leithe-Jasper, H. Rosner, R. Cardoso-Gil, R. Gumeniuk, D. Trots, J. A. Mydosh, and Yu. Grin, Phys. Rev. B 77 (2008) 094421 1-9.

[44] E. Gratz, R. Resel, A. T. Burkov, E. Bauer, A. S. Markosyan and A. Galatanu, J. Phys.: Condens. Matter 7 (1995) 6687-6706.

[45] K. Kadowaki and S. B. Woods, Solid State Commun. 58 (1986) 507-509.

[46] G. P. Meisner, G. R. Stewart, M. S. Torikachvili, and M. B. Maple, Proc LT-17, 1984, pp. 711-712.

[47] T. A. Sayles, R. E. Baumbach, W. M. Yuhasz, M. B. Maple, Ł. Bochenek, R. Wawryk, T. Cichorek, A. Pietraszko, Z. Henkie, and P.-C. Ho, Phys. Rev. B 82 (2010) 104513 1-7.

[48] J. Singleton, Band Theory and Electronic Properties of Solids, Oxford University Press, 2007, p. 133.

[49] R. H. Bube, Electrons in Solids (Academic Press Inc., Boston, 1988) p.172.

[50] K. Behnia, D. Jaccard, and J. Flouquet, J. Phys. :Condens. Matter 16 (2004) 5187-5198.

[51] V. Zlatić, R. Monnier, J. K. Freericks, and K. W. Becker, Phys. Rev. B 76 (2007) 085122 1-16.

[52] F. J. Blatt, P. A. Schroeder, C. I. Foiles, and D. Greig, Thermoelectric Power of Metals (Plenum Press, New York, 1976) p.152.

[53] N. Cusack, The Electrical and Magnetic Properties of Solids (London, 1958) p.116.

[54] N. F. Mott, Phil. Mag. 30 (1974) 403-416. 